\title{
Open Problems Related to the Hurwitz Stability of Polynomials Segments
}

\author{
Baltazar Aguirre-Hernández, ${ }^{1}$ Faustino Ricardo García-Sosa, ${ }^{2}$ \\ Carlos Arturo Loredo-Villalobos $\left(\mathbb{D},{ }^{1,3}\right.$ Raúl Villafuerte-Segura $\left(\mathbb{D},{ }^{4}\right.$ \\ and Eric Campos-Cantón (1D) \\ ${ }^{1}$ Departamento de Matemáticas, Universidad Autónoma Metropolitana-Iztapalapa, San Rafael Atlixco 186, \\ 09340 Ciudad de México, Mexico \\ ${ }^{2}$ SEPI-UPIICSA, Instituto Politécnico Nacional, 08400 Ciudad de México, Mexico \\ ${ }^{3}$ División de Matemáticas Aplicadas, IPICyT, 78216 San Luis, SLP, Mexico \\ ${ }^{4}$ Research Center on Information Technology and Systems, Hidalgo State University, Pachuca, HGO, Mexico \\ ${ }^{5}$ Mathematics Department, University of Houston, Houston, TX 77204-3008, USA
}

Correspondence should be addressed to Carlos Arturo Loredo-Villalobos; calv@xanum.uam.mx

Received 17 July 2017; Accepted 13 December 2017; Published 15 February 2018

Academic Editor: Emilio Turco

Copyright (C) 2018 Baltazar Aguirre-Hernández et al. This is an open access article distributed under the Creative Commons Attribution License, which permits unrestricted use, distribution, and reproduction in any medium, provided the original work is properly cited.

In the framework of robust stability analysis of linear systems, the development of techniques and methods that help to obtain necessary and sufficient conditions to determine stability of convex combinations of polynomials is paramount. In this paper, knowing that Hurwitz polynomials set is not a convex set, a brief overview of some results and open problems concerning the stability of the convex combinations of Hurwitz polynomials is then provided.

\section{Introduction}

The design of control systems arises from the need of human beings to manipulate real plants with some degree of confidence and accuracy. However, this design requires mathematical models which are often very complex if all the dynamics that involve a real plant are taken into account. Thus, a complex model requires a complex controller design. Robust control theory intends to analyze a complex model by studying its linear approximation and always assuming that this approximation will incur with some degree of modeling errors. This error is regarded as uncertainty which is modeled and bounded to determine stability conditions of a system and control laws are obtained.

Problems that arise from robust control theory containing one uncertain parameter, such as gain or constants time, and the system stability should be determined for the entire uncertainty range. The study of criteria for deciding whether a convex combination of polynomials is stable can be applied to resolution of a wide variety of uncertainty systems. The problem to be raised is as follows: find conditions about the stable polynomials $p_{0}(t)$ and $p_{1}(t)$ such that the convex combination described by $p(t, \lambda)=\lambda P_{0}(t)+(1-\lambda) P_{1}(t)$ is stable (Hurwitz), for all $\lambda \in[0,1]$.

The first result, where necessary and sufficient conditions were presented, is known as the Bialas Theorem [1-3]. A different approach in terms of the frequency domain is known as the Segment Lemma and it was found by Bhattacharyya et al. $[4,5]$. This result shows that the stability of $p(t, \lambda)$ is equivalent to certain conditions that must be met by even and odd part of the polynomials $p_{0}(t)$ and $p_{1}(t)$. In complex polynomials case, Bose developed a method to determine the stability of the segment of complex polynomials [6]. In regard to sufficient conditions, in $[7,8]$, the well-known Rantzer's conditions were obtained.

Based on the above criteria computer algorithms have been developed to resolve this problem. The Segment Lemma has been used to design an algorithm in [9]. On this same 
path, a method to verify the stability of convex combinations of polynomials in a finite number of calculus is obtained in [10]. In [11] an algorithm to determine the stability of segment of complex polynomials is proposed; it is based on the work of Bose [12]. In the present paper, we will discuss some of these criteria and propose some open problems in these topics. Applications of Hurwitz stability criterion to Engineering problems are huge, for example, for the stability of wave propagation in strain gradient [13] or mixture [14].

The paper is organized as follows. In Section 2, the problem statement is presented. Some results with necessary and sufficient condition on stability of segment of Hurwitz polynomials and its open problems are presented in Section 3. In Section 4, some open problems with sufficient condition and using Rantzer's conditions are postulated. In Section 5, a related problem with segments of stable polynomials is the calculating of the Minimum Left Extreme, which was studied by Bialas. In Section 6 some results and open problems related to Hadamardized Hurwitz polynomials are postulated. Finally, in Section 7, some concluding remarks are provided.

\section{Problem Statement}

In this section some definitions and one motivation example in the framework of the stability of polynomials are given.

Definition 1. A polynomial with real coefficients, $f(t)=b_{0} t^{n}+$ $b_{1} t^{n-1}+\cdots+b_{n-1} t+b_{n}$, is Hurwitz if all its roots have negative real part.

Definition 2. Suppose that $p_{0}(t)$ and $p_{1}(t)$ are polynomials (real or complex) of degree $n$. Let

$$
P(t, \lambda)=\lambda p_{0}(t)+(1-\lambda) p_{1}(t)
$$

and consider the following one parameter family of polynomials:

$$
\left[p_{0}(t), p_{1}(t)\right]=P(t, \lambda): \quad \lambda \in[0,1]
$$

This family will be referred to as a segment of polynomials. We shall say that the segment is stable if and only if every polynomial on the segment is stable.

The following example shows that a segment of polynomials is not necessarily a stable segment although the two extremes are Hurwitz polynomials.

Example 3. Let $P_{0}(t)=t^{3}+6 t^{2}+11 t+6=(t+1)(t+2)(t+3)$ and $P_{1}(t)=6 t^{2}+5 t+216$ be Hurwitz; nevertheless

$$
\begin{aligned}
& \lambda P_{0}(t)+(1-\lambda) P_{1}(t) \\
& \quad=\lambda\left(t^{3}+6 t^{2}+11 t+6 t\right)+(1-\lambda)\left(6 t^{2}+5 t+216\right)
\end{aligned}
$$

is not Hurwitz for all $\lambda \geq 0$ :

(i) for $\lambda \in[0,(5-\sqrt{2}) / 7)$ it is Hurwitz;

(ii) for $\lambda \in[(5-\sqrt{2}) / 7,(5+\sqrt{2}) / 7]$ it is not Hurwitz;

(iii) for $\lambda \in((5+\sqrt{2}) / 7,1]$ it is Hurwitz.

Now, there are several criteria to determine the stability of the segment of polynomials; we present some of them below.

\section{Necessary and Sufficient Conditions}

In this section, we present two of the most known necessary and sufficient conditions on the stability of segment of Hurwitz polynomials.

\subsection{The Bialas Theorem}

Theorem 4 (see [2]). If $p_{0}$ is Hurwitz and $\operatorname{deg}\left(p_{0}\right)>\operatorname{deg}\left(p_{1}\right)$, then $P(t, \lambda)=\lambda p_{0}(t)+(1-\lambda) p_{1}(t)$ is Hurwitz for all $\lambda \in[0,1]$, if and only if the matrix $H^{-1}\left(p_{0}\right) H\left(p_{1}\right)$ has eigenvalues not in $(-\infty, 0)$, where $H^{-1}\left(p_{0}\right)$ and $H\left(p_{1}\right)$ are matrices Hurwitz of $p_{0}$ and $p_{1}$, respectively.

Open Problem 1. Is it possible to find necessary and sufficient conditions in a similar way of the Bialas Theorem for $\operatorname{deg}\left(p_{0}\right)=\operatorname{deg}\left(p_{1}\right)$ case?

In order to establish the Segment Lemma we give some definitions.

Given a polynomial $p(t)=a_{0}+a_{1} t+a_{2} t^{2}+\cdots+a_{n} t^{n}$, we define

$$
\begin{aligned}
& p^{\text {even }}(s)=a_{0}+a_{2} t^{2}+\cdots, \\
& p^{\text {odd }}(s)=a_{1} t+a_{3} t^{3}+\cdots,
\end{aligned}
$$

then $p(t)=p^{\text {even }}(t)+p^{\text {odd }}(t)$ and $p(i \omega)$ can be written as $p(i \omega)=p^{e}(\omega)+i \omega p^{o}(\omega)$, where

$$
\begin{aligned}
& p^{e}(\omega)=a_{0}-a_{2} \omega^{2}+a_{4} \omega^{4}+\cdots, \\
& p^{o}(\omega)=a_{1}-a_{3} \omega^{2}+a_{5} \omega^{4}+\cdots .
\end{aligned}
$$

In the Bialas Theorem, it is necessary that the two extremes of the segment of polynomials have different degree. Now we present the Segment Lemma, where the two extremes of the segment of polynomials have equal degrees.

\subsection{The Segment Lemma}

Lemma 5 (see $[4,5]$ ). Let $p_{1}(t)$ and $p_{2}(t)$ be $n$-degree Hurwitz polynomials with leading coefficients of the same sign. Then the segment of polynomials $\left[p_{1}(t), p_{2}(t)\right]$ is Hurwitz, if and only if there are not $\omega>0$ that satisfies the three conditions:

(1) $p_{1}^{e}(\omega) p_{2}^{o}(\omega)-p_{2}^{e}(\omega) p_{1}^{o}(\omega)=0$,

(2) $p_{1}^{e}(\omega) p_{2}^{e}(\omega) \leq 0$,

(3) $p_{1}^{o}(\omega) p_{2}^{o}(\omega) \leq 0$. 
Open Problem 2. Is it possible to get a result, based on the approach of the Segment Lemma, to be applicable when polynomials $p_{0}$ and $p_{1}$ have different degrees?

After we have discussed two necessary and sufficient conditions, now in the next section, we expose two sufficient conditions: Rantzer's conditions and one approach of matrix inequalities.

\section{Sufficient Conditions}

In this section, we present two sufficient conditions for the stability of segment that are known in the literature: Rantzer's conditions and one interesting approach with matrix inequalities.

4.1. Rantzer's Conditions. Supposing that $p_{0}(t)$ is a Hurwitz polynomial and $P_{1}(t)$ is semistable (their roots have real part $0)$, then the segment of polynomials $\left[p_{0}(t), p_{1}(t)\right]$ consists of Hurwitz polynomials if you have one of the following four conditions.

(i) The difference $d=p_{1}-p_{0}$ satisfies

$$
\frac{\partial \arg (d(i \omega))}{\partial \omega}<0, \quad \omega \in\{w>0 \mid d(i w) \neq 0\} .
$$

(ii) Each of the polynomials $p_{0}$ and $p_{1}$ has at least one root in $\mathbb{C}^{-}$and

$$
\begin{array}{r}
\frac{\partial \arg (d(i \omega))}{\partial \omega}<\left|\frac{\sin (2 \arg [d(i \omega)])}{2 \omega}\right|, \\
\quad \omega \in\{w>0 \mid d(i w) \neq 0\} .
\end{array}
$$

(iii) Each of the polynomials $p_{0}$ and $p_{1}$ has at least one root in $\mathbb{C}^{-}$and

$$
\frac{\partial \arg (d(i \omega))}{\partial \omega} \leq 0, \quad \omega \in\{w>0 \mid d(i w) \neq 0\} .
$$

(iv) Each of the polynomials $p_{0}$ and $p_{1}$ has at least two roots in $\mathbb{C}^{-}$and

$$
\begin{array}{r}
\frac{\partial \arg (d(i \omega))}{\partial \omega} \leq\left|\frac{\sin (2 \arg [d(i \omega)])}{2 \omega}\right| \\
\omega \in\{w>0 \mid d(i w) \neq 0\} .
\end{array}
$$

The previous conditions are known as Rantzer's conditions; see [8].

Open Problem 3. Is there a mechanism to describe Rantzer's conditions in terms of the polynomial coefficients?

Open Problem 4. What should we add to Rantzer's conditions in order to obtain necessary and sufficient conditions?

In the next subsection, we present an interesting sufficient condition for checking the stability of segment of polynomials based on matrix inequalities.
4.2. A Matrix Inequality. Given a polynomial $p_{0}(t)=t^{n}+$ $a_{1} t^{n-1}+\cdots+a_{n}$, we define the matrix $E_{(n, n)} \in \mathscr{M}_{(n+1) \times(n+1)}$ as

$$
E_{(n, n)}=\left(\begin{array}{ccccccc}
1 & 0 & 0 & 0 & \cdots & 0 & 0 \\
-a_{2} & a_{1} & -1 & 0 & \cdots & 0 & 0 \\
a_{4} & -a_{3} & a_{2} & -a_{1} & \cdots & 0 & 0 \\
\cdots & \cdots & \cdots & \cdots & \cdots & \cdots & \cdots \\
0 & 0 & 0 & 0 & \cdots & a_{n-1} & -a_{n-2} \\
0 & 0 & 0 & 0 & \cdots & 0 & a_{n}
\end{array}\right) .
$$

Theorem 6. Let $p_{1}=c_{1} t^{n}+c_{2} t^{n-1}+\cdots+c_{n+1}$ be a polynomial of degree $n$. If the polynomials $p_{0}(t)$ and $p_{1}(t)$ are Hurwitz and vector $c=\left(c_{1}, c_{2}, \ldots, c_{n+1}\right)^{T} \geq 0$ satisfies the system of linear inequalities,

$$
E_{(n, n)} \mathcal{c} \supsetneqq 0,
$$

then $\lambda p_{0}(t)+(1-\lambda) p_{1}(t)$ is Hurwitz for all $\lambda \in[0,1]$. Here the symbol $\geq 0(\leq 0)$ means that all components of a given vector are $\geq 0(\leq 0)$ and the symbol $¥ 0$ means that all components of a given vector are $\geq 0$ but there is at least one component which is $>0$. See $[15,16]$.

Open Problem 5. With these approach matrix inequalities, what should we add to obtain necessary and sufficient conditions?

4.2.1. Case for Grade $p_{1}=n-1$. A similar case is obtained if $\operatorname{deg}\left(p_{1}(t)\right)=n-1$. In this case, the matrix $E_{(n, n-1)} \in \mathscr{M}_{(n+1) \times n}$ is defined by

$$
\begin{aligned}
& E_{(n, n-1)} \\
& \quad=\left(\begin{array}{ccccccc}
a_{1} & -1 & 0 & 0 & \cdots & 0 & 0 \\
-a_{3} & a_{2} & -a_{1} & 1 & \cdots & 0 & 0 \\
a_{5} & -a_{4} & a_{3} & -a_{2} & \cdots & 0 & 0 \\
\cdots & \cdots & \cdots & \cdots & \cdots & \cdots & \cdots \\
0 & 0 & 0 & 0 & \cdots & a_{n-1} & -a_{n-2} \\
0 & 0 & 0 & 0 & \cdots & 0 & a_{n}
\end{array}\right),
\end{aligned}
$$

and the corresponding inequality is

$$
E_{(n, n-1)} c \supsetneqq 0 .
$$

There is a relation between segments and rays of polynomials, which let us give applications in control theory. We explain it in the next two subsections.

4.3. Relationship between Rays and Segment of Polynomials. If $p_{0}(t)+k p_{1}(t)$ is Hurwitz for all $k \geq 0$, then $(1 /(1+k)) p_{0}(t)+$ $(k /(1+k)) p_{1}(t)$ is Hurwitz for all $k \geq 0$, from which if $p_{1}$ is Hurwitz, then, the stability of ray $p_{0}(t)+k p_{1}(t)$ is equivalent to the stability of the segment $\left[p_{0}(t), p_{1}(t)\right]$. An analysis in terms of ray polynomials was made in [15]. 
For $\operatorname{deg}\left(p_{1}(t)\right)=n, D_{(n, n)} \in \mathscr{M}_{n \times(n+1)}$ is the matrix:

$$
\begin{aligned}
& D_{(n, n)} \\
& \quad=\left(\begin{array}{ccccccc}
a_{1} & -1 & 0 & 0 & \cdots & 0 & 0 \\
-a_{3} & a_{2} & -a_{1} & 1 & \cdots & 0 & 0 \\
a_{5} & -a_{4} & a_{3} & -a_{2} & \cdots & 0 & 0 \\
\cdots & \cdots & \cdots & \cdots & \cdots & \cdots & \cdots \\
0 & 0 & 0 & 0 & \cdots & -a_{n-2} & a_{n-3} \\
0 & 0 & 0 & 0 & \cdots & a_{n} & -a_{n-1}
\end{array}\right)
\end{aligned}
$$

for $\operatorname{deg}\left(p_{1}(t)\right)=n-1, D_{(n, n-1)} \in \mathscr{M}_{n \times n}$ is the matrix:

$$
\begin{aligned}
& D_{(n, n-1)} \\
& \quad=\left(\begin{array}{ccccccc}
1 & 0 & 0 & 0 & \cdots & 0 & 0 \\
-a_{2} & a_{1} & -1 & 0 & \cdots & 0 & 0 \\
a_{4} & -a_{3} & a_{2} & -a_{1} & \cdots & 0 & 0 \\
\cdots & \cdots & \cdots & \cdots & \cdots & \cdots & \cdots \\
0 & 0 & 0 & 0 & \cdots & a_{n-2} & -a_{n-3} \\
0 & 0 & 0 & 0 & \cdots & -a_{n} & a_{n-1}
\end{array}\right) ;
\end{aligned}
$$

for $\operatorname{deg}\left(p_{1}(t)\right)=n-2, D_{(n, n-2)} \in \mathscr{M}_{(n-1) \times(n-1)}$ is the matrix:

$$
\begin{aligned}
& D_{(n, n-2)} \\
& \quad=\left(\begin{array}{ccccccc}
a_{1} & -1 & 0 & 0 & \cdots & 0 & 0 \\
-a_{3} & a_{2} & -a_{1} & 1 & \cdots & 0 & 0 \\
a_{5} & -a_{4} & a_{3} & -a_{2} & \cdots & 0 & 0 \\
\cdots & \cdots & \cdots & \cdots & \cdots & \cdots & \cdots \\
0 & 0 & 0 & 0 & \cdots & a_{n-2} & -a_{n-3} \\
0 & 0 & 0 & 0 & \cdots & -a_{n} & a_{n-1}
\end{array}\right) .
\end{aligned}
$$

Rewriting the results shown in $[15,16]$ in terms of segment polynomials yields the following result.

Theorem 7. Consider the Hurwitz polynomial $p_{0}(t)=t^{n}+$ $a_{1} t^{n-1}+\cdots+a_{n}$. If $p_{1}(t)$ is Hurwitz with $\operatorname{deg}\left(p_{1}(t)\right)=n, n-1$ or $n-2$ and the vector of coefficients $c$ satisfies the system of linear inequalities

$$
D c \supsetneqq 0 ;
$$

then the polynomial $\lambda p_{0}(t)+(1-\lambda) p_{1}(t)$ is Hurwitz for all $\lambda \in[0,1]$; where the matrix $D$ is determined by the degree of $p_{1}(t)$ and is one of the matrices $D_{(n, n)}, D_{(n, n-1)}$, or $D_{(n, n-2)}$.

Example 8. Consider the polynomial Hurwitz $p_{0}(t)=t^{3}+$ $(1 / 12) t^{2}+5 t+1 / 12$. The vector of coefficients $c$ of polynomial $p_{1}(t)=t^{3}+37 t^{2}+(11 / 2) t+1$ is solution of system of linear inequalities (11):

$$
\begin{aligned}
E_{(3,3)} c & =\left(\begin{array}{cccc}
1 & 0 & 0 & 0 \\
-5 & \frac{1}{12} & -1 & 0 \\
0 & -\frac{1}{12} & 5 & -\frac{1}{12} \\
0 & 0 & 0 & \frac{1}{12}
\end{array}\right)\left(\begin{array}{c}
1 \\
37 \\
\frac{11}{2} \\
1
\end{array}\right) \\
& =\left(\begin{array}{c}
1 \\
8 \\
\frac{17}{2} \\
\frac{1}{12}
\end{array}\right) ;
\end{aligned}
$$

therefore, the segment polynomial $\left[p_{0}, p_{1}\right]$ is Hurwitz. However, $c$ is not a solution of (17):

$$
\begin{aligned}
D_{(3,3)} c & =\left(\begin{array}{cccc}
\frac{1}{2} & -1 & 0 & 0 \\
-\frac{1}{2} & 5 & -\frac{1}{2} & 1 \\
0 & 0 & \frac{1}{2} & -5
\end{array}\right)\left(\begin{array}{c}
1 \\
37 \\
\frac{11}{2} \\
1
\end{array}\right) \\
& =\left(\begin{array}{c}
-\frac{73}{2} \\
\frac{731}{4} \\
-\frac{9}{4}
\end{array}\right),
\end{aligned}
$$

and Rantzer's conditions are not verified: to the difference $d=$ $p_{1}-p_{0}=(73 / 2) t^{2}+(1 / 12) t+1 / 12$ has

$$
\begin{aligned}
& \frac{\partial \arg (d(i \omega))}{\partial \omega}=\frac{1 / 14+(73 / 4) \omega^{2}}{\left[1 / 12-(73 / 2) \omega^{2}\right]^{2}+(1 / 12) \omega^{2}} \\
& >0, \\
& \frac{\sin (2 \arg [d(i \omega)])}{2 \omega} \\
& =\frac{1 / 14-(73 / 4) \omega^{2}}{\left[1 / 12-(73 / 2) \omega^{2}\right]^{2}+(1 / 12) \omega^{2}} .
\end{aligned}
$$

Open Problem 6. Will there be a stable segment of polynomial that satisfies Rantzer's conditions but does not satisfy (11) or (17)?

The importance of the segments and rays of Hurwitz polynomials with the design of stabilizing controls is pointed out in the next subsection. 
4.4. Applications to Control Systems. Consider the system $\dot{x}=$ $A x+b u$, where

$$
\begin{aligned}
A & =\left[\begin{array}{ccccc}
0 & 1 & 0 & \cdots & 0 \\
0 & 0 & 1 & \cdots & 0 \\
\vdots & \vdots & \vdots & & \vdots \\
0 & 0 & 0 & \cdots & 1 \\
-a_{n} & -a_{n-1} & -a_{n-2} & \cdots & -a_{1}
\end{array}\right], \\
b & =\left[\begin{array}{c}
0 \\
0 \\
\vdots \\
0 \\
1
\end{array}\right] .
\end{aligned}
$$

For the uncontrolled system $\dot{x}=A x$, the characteristic polynomial is $p_{0}(t)=t^{n}+a_{1} t^{n-1}+a_{2} t^{n-2}+\cdots+a_{n}$. If into the controlled system $\dot{x}=A x+b u$ we choose a control $u$ as $u(x)=-k c^{T} x=-k\left(c_{n}, c_{n-1}, \ldots, c_{1}\right) x$, where $k>0$ and $c \in \mathbb{R}^{n}$, then the controlled system takes the form:

$$
\dot{x}=\left(A-k b c^{T}\right) x,
$$

and its characteristic polynomial is

$$
\begin{aligned}
p(t)= & t^{n}+a_{1} t^{n-1}+a_{2} t^{n-2}+\cdots+a_{n} \\
& +k\left[c_{1} t^{n-1}+c_{2} t^{n-2}+\cdots+c_{n}\right],
\end{aligned}
$$

that is,

$$
p(t)=p_{0}(t)+k p_{1}(t)
$$

where $p_{1}(t)=c_{1} t^{n-1}+c_{2} t^{n-2}+\cdots+c_{n}$.

Example 9. Consider the system

$$
\dot{x}=\left(\begin{array}{ccc}
0 & 1 & 0 \\
0 & 0 & 1 \\
-1 & -\frac{1}{32} & -2
\end{array}\right) x+\left(\begin{array}{l}
0 \\
0 \\
1
\end{array}\right)(-2 k,-4 k,-2 k) x .
$$

In this case, the matrix $E$ is given by

$$
E=\left(\begin{array}{ccc}
2 & -1 & 0 \\
-1 & \frac{3}{2} & -2 \\
0 & 0 & 1
\end{array}\right)
$$

then we have the system

$$
\left(\begin{array}{ccc}
2 & -1 & 0 \\
-1 & \frac{3}{2} & -2 \\
0 & 0 & 1
\end{array}\right)\left(\begin{array}{l}
2 \\
4 \\
2
\end{array}\right)=\left(\begin{array}{l}
0 \\
0 \\
2
\end{array}\right)
$$

Therefore, the system is stable $\forall k \geq 0$. But this cannot be verified with the results presented by Aguirre et al. in [15].

$$
\begin{aligned}
& D=\left(\begin{array}{ccc}
1 & 0 & 0 \\
-\frac{3}{2} & 2 & -1 \\
0 & -1 & \frac{3}{2}
\end{array}\right), \\
&\left(\begin{array}{ccc}
1 & 0 & 0 \\
-\frac{3}{2} & 2 & -1 \\
0 & -1 & \frac{3}{2}
\end{array}\right)\left(\begin{array}{l}
2 \\
4 \\
2
\end{array}\right)=\left(\begin{array}{c}
2 \\
3 \\
-1
\end{array}\right) .
\end{aligned}
$$

The Minimum Left Extreme is a subject very related to the study of segment of Hurwitz polynomials; hence in the next sections we present some ideas about the Minimum Left Extreme in order to propose some open problems.

\section{The Minimum Left Extreme}

A related problem with segment of Hurwitz polynomials is the calculating of the Minimum Left Extreme, which was studied by Bialas. We begin with some definitions.

Let $p_{0}(t)$ be a Hurwitz polynomial that generates a family of polynomials given as follows:

$$
p(t)=p_{0}(t)+k p_{1}(t)
$$

where $k \in \mathbb{R}$ and $p_{1}(t)=\sum_{i=1}^{n} c_{i} t^{n-i}$ is a polynomial with coefficient vector $c=\left(c_{1}, c_{2}, \ldots, c_{n}\right)^{T} \succeq 0$. If $E_{(n, n-1)}$ is the matrix defined in the previous section and $c$ is a solution of the system of linear inequalities $E_{(n, n-1)} c \supsetneqq 0$, then $p_{0}(t)+$ $k p_{1}(t)$ is a Hurwitz polynomial $\forall k \geq 0$.

What is the value of $k_{\min }<0$, so that $p_{0}(t)+k p_{1}(t)$ is Hurwitz $\forall k \geq k_{\min }$ ? From Bialas (see [2]),

$$
k_{\min }=\frac{1}{\lambda_{\text {min }}^{-}\left[-H^{-1}\left(p_{0}\right) H\left(p_{1}\right)\right]},
$$

where $H\left(p_{0}\right)$ and $H\left(p_{1}\right)$ are Hurwitz matrices of $p_{0}$ and $p_{1}$, respectively, and $\lambda_{\text {min }}^{-}\left[-H^{-1}\left(p_{0}\right) H\left(p_{1}\right)\right]$ is the smallest negative eigenvalue of the matrix

$$
-H^{-1}\left(p_{0}\right) H\left(p_{1}\right)
$$

5.1. Estimation Technique Proposed. Consider the polynomial $p_{0}(t)=t^{n}+a_{1} t^{n-1}+\cdots+a_{n}$ whereby the following matrix is defined:

$$
\begin{aligned}
& Z_{(n, n-1)} \\
& \quad=\left(\begin{array}{ccccccc}
a_{1} & -2 & 0 & 0 & \cdots & 0 & 0 \\
0 & a_{2} & -2 a_{1} & 2 & \cdots & 0 & 0 \\
0 & 0 & a_{3} & -2 a_{2} & \cdots & 0 & 0 \\
\cdots & \cdots & \cdots & \cdots & \cdots & \cdots & \cdots \\
0 & 0 & 0 & 0 & \cdots & a_{n-1} & -2 a_{n-2} \\
0 & 0 & 0 & 0 & \cdots & 0 & a_{n}
\end{array}\right),
\end{aligned}
$$


with $Z_{(n, n-1)}^{i}$ the $i$ th row of the matrix $Z_{(n \cdot n-1)}$ and $a=$ $\left(a_{1}, a_{2}, \ldots, a_{n}\right)^{T}$.

Theorem 10. Let $p_{0}(t)=t^{n}+a_{1} t^{n-1}+\cdots+a_{n}$ and $E_{(n, n-1)}$ be a Hurwitz polynomial and a matrix defined above, respectively. If the vector $c=\left(c_{1}, c_{2}, \ldots, c_{n}\right)^{T} \geq 0$ is a solution to the system of linear inequalities $E_{(n, n-1)} c \supsetneqq 0$ and $p_{1}(t)=$ $\sum_{i=1}^{n} c_{i} t^{n-i}$, then $p_{0}(t)+k p_{1}(t)$ is Hurwitz $\forall k>k_{0}$, where $k_{0}=$ $\max _{i=1, \ldots, n}\left(-\left(Z_{(n, n-1)}^{i} a / E_{(n, n-1)}^{i} c\right)\right)$; that is, $k_{\min } \leq k_{0}$.

Example 11. Given $p_{0}(t)=t^{3}+7 t^{2}+14 t+8$ and $p_{1}(t)=$ $t^{2}+4 t+6$, we have that

$$
\begin{aligned}
& Z_{(3,2)} a=\left(\begin{array}{ccc}
7 & -2 & 0 \\
0 & 14 & -14 \\
0 & 0 & 8
\end{array}\right)\left(\begin{array}{c}
7 \\
14 \\
8
\end{array}\right) \\
& =\left(\begin{array}{l}
21 \\
84 \\
64
\end{array}\right) \text {, } \\
& E_{(3,2)} \mathcal{c}=\left(\begin{array}{ccc}
7 & -1 & 0 \\
-8 & 14 & -7 \\
0 & 0 & 8
\end{array}\right)\left(\begin{array}{l}
1 \\
4 \\
6
\end{array}\right)=\left(\begin{array}{c}
3 \\
6 \\
48
\end{array}\right), \\
& k_{0}=\max \left(-\frac{21}{3},-\frac{84}{6},-\frac{64}{48}\right)=-\frac{1}{43} \text {, } \\
& H\left(p_{0}\right)=\left(\begin{array}{ccc}
7 & 8 & 0 \\
1 & 14 & 0 \\
0 & 7 & 8
\end{array}\right) \text {, } \\
& H\left(p_{1}\right)=\left(\begin{array}{lll}
1 & 6 & 0 \\
0 & 4 & 0 \\
0 & 1 & 6
\end{array}\right), \\
& H^{-1}\left(p_{0}\right) H\left(p_{1}\right)=\left(\begin{array}{rrr}
\frac{7}{45} & \frac{26}{45} & 0 \\
-\frac{1}{90} & \frac{11}{45} & 0 \\
\frac{7}{720} & -\frac{4}{45} & \frac{3}{4}
\end{array}\right) \text {. }
\end{aligned}
$$

Then $\sigma\left(-H^{-1}\left(p_{0}\right) H\left(p_{1}\right)\right)=\{-1 / 34,-1 / 15 \pm(1 / 15) i\}$. We have that $\lambda_{\min }=-1 / 34, k_{\min }=-1 / 43$.

In the above example, the two approaches lead to $p_{0}(t)+$ $k p_{1}(t)$ being Hurwitz $\forall k>-1 / 43$.

Example 12. Given $p_{0}(t)=t^{3}+7 t^{2}+14 t+8$ and $p_{1}(t)=$ $26 t^{2}+137 t+90$, we have that

$$
Z_{(3,2)} a=\left(\begin{array}{ccc}
7 & -2 & 0 \\
0 & 14 & -14 \\
0 & 0 & 8
\end{array}\right)\left(\begin{array}{c}
7 \\
14 \\
8
\end{array}\right)
$$

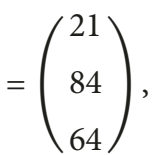$$
E_{(3,2)} \mathcal{c}=\left(\begin{array}{ccc}
7 & -1 & 0 \\
-8 & 14 & -7 \\
0 & 0 & 8
\end{array}\right)\left(\begin{array}{c}
26 \\
137 \\
90
\end{array}\right)
$$$$
=\left(\begin{array}{c}
45 \\
1080 \\
720
\end{array}\right) \text {, }
$$$$
k_{0}=\max \left(-\frac{21}{45},-\frac{84}{1080},-\frac{64}{720}\right)
$$$$
=-\frac{7}{90}=-0.07778 \text {, }
$$$$
H\left(p_{0}\right)=\left(\begin{array}{ccc}
7 & 8 & 0 \\
1 & 14 & 0 \\
0 & 7 & 8
\end{array}\right),
$$$$
H\left(p_{1}\right)=\left(\begin{array}{ccc}
26 & 90 & 0 \\
0 & 137 & 0 \\
0 & 26 & 90
\end{array}\right) \text {, }
$$

$$
H^{-1}\left(p_{0}\right) H\left(p_{1}\right)=\left(\begin{array}{ccc}
\frac{182}{45} & \frac{82}{45} & 0 \\
-\frac{13}{45} & \frac{869}{90} & 0 \\
\frac{91}{360} & -\frac{3743}{720} & \frac{45}{4}
\end{array}\right) \text {. }
$$

Then $\sigma\left(-H^{-1}\left(p_{0}\right) H\left(p_{1}\right)\right)=\{-11.25,-4.1399,-9.5601\}$. Finally we have $\lambda_{\min }=-11.25, k_{\min }=-0.088889$.

Now, in Example 12 with the approach proposed in Theorem 10, we obtain that $p_{0}(t)+k p_{1}(t)$ is Hurwitz $\forall k>$ -0.07778 and applying the Bialas approach we obtain that $p_{0}(t)+k p_{1}(t)$ is Hurwitz $\forall k>-0.088889$. That is, $k_{\min }<k_{0}$.

Example 13. Consider the following system:

$$
\begin{aligned}
\dot{x}= & \left(\begin{array}{ccc}
0 & 1 & 0 \\
0 & 0 & 1 \\
-8 & -14 & -7
\end{array}\right) x \\
& +\left(\begin{array}{l}
0 \\
0 \\
1
\end{array}\right)(-90 k,-137 k,-26) x .
\end{aligned}
$$

Here $p_{0}(t)=t^{3}+7 t^{2}+14 t+8$ and $p_{1}(t)=26 t^{2}+137 t+90$. Then it holds 


$$
\begin{aligned}
k_{0} & =\max \left(-\frac{21}{45},-\frac{84}{1080},-\frac{64}{720}\right)=-\frac{7}{90}=-0.07778 \\
& >-1
\end{aligned}
$$

Therefore, in addition, we can ensure that the system is stable for all $k>0$ (the control $u$ is a stabilizing control) and we can also say more, the system is stable for all $k>-0.07778$ because $p_{0}(t)+k p_{1}(t)$ is Hurwitz $\forall k>-0.07778$.

Open Problem 7. Describe the cases when $k_{0}=k_{\min }$.

Remark 14. An advantage of our approach, in relation to the Bialas approach, is that we can estimate the Minimum Left Extreme when the polynomials have the same degree. Given the polynomial $p_{0}(t)=t^{n}+a_{1} t^{n-1}+\cdots+a_{n}$, the matrices $Z_{(n, n)}$ and $E_{(n, n)}$ are defined by

$$
\begin{gathered}
Z_{(n, n)}=\left(\begin{array}{ccccccc}
1 & 0 & 0 & 0 & \cdots & 0 & 0 \\
0 & a_{1} & -2 & 0 & \cdots & 0 & 0 \\
0 & 0 & a_{2} & -2 a_{1} & \cdots & 0 & 0 \\
\cdots & \cdots & \cdots & \cdots & \cdots & \cdots & \cdots \\
0 & 0 & 0 & 0 & \cdots & a_{n-1} & -2 a_{n-2} \\
0 & 0 & 0 & 0 & \cdots & 0 & a_{n}
\end{array}\right), \\
E_{(n, n)}= \\
\left.\begin{array}{ccccccc}
1 & 0 & 0 & 0 & \cdots & 0 & 0 \\
-a_{2} & a_{1} & -1 & 0 & \cdots & 0 & 0 \\
a_{4} & -a_{3} & a_{2} & -a_{1} & \cdots & 0 & 0 \\
\cdots & \cdots & \cdots & \cdots & \cdots & \cdots & \cdots \\
0 & 0 & 0 & 0 & \cdots & a_{n-1} & -a_{n-2} \\
0 & 0 & 0 & 0 & \cdots & 0 & a_{n}
\end{array}\right)
\end{gathered}
$$

If the vector $c=\left(c_{0}, c_{1}, \ldots, c_{n}\right)^{T} \succeq 0$ is a solution of inequalities system $E_{(n, n)} c \supsetneqq 0$ and the polynomial $p_{1}(t)$ is given by $p_{1}(t)=\sum_{i=0}^{n} c_{i} t^{n-i}$, then $p_{0}(t)+k p_{1}(t)$ is a Hurwitz polynomial $\forall k>k_{0}$, where $k_{0}=\max _{i=1, \ldots, n, n+1}\left(-Z^{i} a / E^{i} c\right)$; that is, $k_{\min } \leq k_{0}$.

Open Problem 8. With this approach find conditions for calculating $k_{0}$ as $\operatorname{deg}\left(p_{0}\right)>\operatorname{deg}\left(p_{1}\right)$ or as $\operatorname{deg}\left(p_{0}\right)=\operatorname{deg}\left(p_{1}\right)$.

If we consider the set of the Hadamardized Hurwitz polynomials, then we can plant some open problems also. It is the motivation to include the next section.

\section{Hadamardized Hurwitz Polynomials}

In the present section, some results and open problems related to Hadamardized Hurwitz polynomials are postulated.

Another property of Hurwitz polynomials is related to Hadamard product. Consider two real polynomials $p(t)$ and $q(t)$ :

$$
\begin{aligned}
& p(t)=a_{n} t^{n}+a_{n-1} t^{n-1}+\cdots+a_{1} t+a_{0}, \\
& q(t)=b_{n} t^{n}+b_{n-1} t^{n-1}+\cdots+b_{1} t+b_{0} .
\end{aligned}
$$

The Hadamard product of $p(t)$ and $q(t)$, denoted by $(p * q)(t)$, is defined by

$$
\begin{aligned}
(p * q)(t)= & a_{n} b_{n} t^{n}+a_{n-1} b_{n-1} t^{n-1}+\cdots+a_{1} b_{1} t \\
& +a_{0} b_{0} t .
\end{aligned}
$$

In [17], it was proved that the set of Hurwitz polynomials is closed under Hadamard product. However in [18] it was shown that there are Hurwitz polynomials of degree 4 that do not have a Hadamard factorization in two Hurwitz polynomials. In $[19,20]$, some conditions to Hadamard factorization existence were presented. The set of Hurwitz polynomials that admits a Hadamard factorization, we called Hadamardized Hurwitz polynomials, is denoted by $\mathscr{H}(\mathrm{Had})_{n}$. Recently, in [21] some topological properties about this set were shown. But regarding the convex combination, we can propose the following problems.

Open Problem 9. Given $f, g \in \mathscr{H}(\mathrm{Had})_{n}$, what conditions are required to guarantee that $\lambda f+(1-\lambda) g \in \mathscr{H}(\mathrm{Had})_{n}$ ?

Open Problem 10. Is there any property in $H_{n}$ set that does not share with $\mathscr{H}(\mathrm{Had})_{n}$ set?

\section{Conclusions}

In this paper, a brief overview of some results concerning the stability of the convex combination of Hurwitz polynomials is provided. Also some criteria for deciding if a segment of polynomials is a Hurwitz polynomial were presented. Furthermore some open problems about these polynomials in order to motivate the study of them were given. As mentioned above, the study and solution of these problems also have their field of opportunity in the framework of robust systems.

\section{Conflicts of Interest}

The authors declare that there are no conflicts of interest regarding the publication of this paper.

\section{Acknowledgments}

This work is part of the sabbatical year by Faustino Ricardo García-Sosa realized at Universidad Autónoma del Estado de Hidalgo, Mexico. The author Carlos Arturo LoredoVillalobos would like to thank the support of CONACYT through the postdoctoral fellowship.

\section{References}

[1] B. R. Barmish, New Tools for Robustness of Linear Systems, McMillan, 1994.

[2] S. Bialas, "A necessary and sufficient condition for the stability of convex combinations of stable polynomials or matrices," Bulletin of the Polish Academy of Sciences-Technical Sciences, vol. 33, no. 9-10, pp. 473-480, 1985. 
[3] M. Fu and B. R. Barmish, "Maximal unidirectional perturbation bounds for stability of polynomials and matrices", Systems \& Control Letters, vol. 11, no. 3, pp. 173-179, 1988.

[4] S. P. Bhattacharyya, H. Chapellat, and L. H. Keel, Robust Control: The Parametric Approach, Prentice-Hall, 1995.

[5] H. Chapellat and S. P. Bhattacharyya, "An alternative proof of Kharitonov's theorem," Institute of Electrical and Electronics Engineers Transactions on Automatic Control, vol. 34, no. 4, pp. 448-450, 1989.

[6] N. K. Bose, "Tests for Hurwitz and Schur Properties of Convex Combination of Complex Polynomials," IEEE Transactions on Circuits and Systems II: Express Briefs, vol. 36, no. 9, pp. 12451247, 1989.

[7] D. Hinrichsen and V. L. Kharitonov, "Stability of polynomials with conic uncertainty," Mathematics of Control, Signals, and Systems, vol. 8, no. 2, pp. 97-117, 1995.

[8] A. Rantzer, "Stability conditions for polytopes of polynomials," Institute of Electrical and Electronics Engineers Transactions on Automatic Control, vol. 37, no. 1, pp. 79-89, 1992.

[9] H. Bouguerra, B. C. Chang, H. H. Yeh, and S. S. Banda, "Fast Stability Checking for the Convex Combination of Stable Polynomials," IEEE Transactions on Automatic Control, vol. 35, no. 5, pp. 586-588, 1990.

[10] C. Hwang and S.-F. Yang, "Use of Routh array for testing the Hurwitz property of a segment of polynomials," Automatica, vol. 37, no. 2, pp. 291-296, 2001.

[11] B. S. Bollepalli and L. R. Pujara, "On the Stability of a Segment of Polynomials," IEEE Transactions on Circuits and Systems I: Fundamental Theory and Applications, vol. 41, no. 12, pp. 898901, 1994.

[12] N. K. Bose, "Argument conditions for Hurwitz and Schur polynomials from network theory," Institute of Electrical and Electronics Engineers Transactions on Automatic Control, vol. 39, no. 2, pp. 345-346, 1994.

[13] F. dell'Isola, A. Madeo, and L. Placidi, "Linear plane wave propagation and normal transmission and reflection at discontinuity surfaces in second gradient 3D continua," Zeitschrift fur Angewandte Mathematik und Mechanik, vol. 92, no. 1, pp. 52-71, 2012.

[14] L. Placidi, F. Dell'Isola, N. Ianiro, and G. Sciarra, "Variational formulation of pre-stressed solid-fluid mixture theory, with an application to wave phenomena," European Journal of Mechanics - A/Solids, vol. 27, no. 4, pp. 582-606, 2008.

[15] B. Aguirre, C. Ibarra, and R. Suárez, "Sufficient algebraic conditions for stability of cones of polynomials," Systems \& Control Letters, vol. 46, no. 4, pp. 255-263, 2002.

[16] B. Aguirre and R. Suárez, "Algebraic test for the Hurwitz stability of a given segment of polynomials," Boletín de la Sociedad Matemática Mexicana, Serie 3, vol. 12, pp. 263-277, 2006.

[17] J. Garloff and D. G. Wagner, "Hadamard products of stable polynomials are stable," Journal of Mathematical Analysis and Applications, vol. 202, no. 3, pp. 797-809, 1996.

[18] J. Garloff and B. Srinivasan, "The Hadamard factorization of HURwitz and Schur stable polynomials," in Stability Theory, R. Jeltsch and M. Mansour, Eds., vol. 121, pp. 19-21, Birkhäuser, Berlin, Germany, 1996.

[19] C. A. Loredo-Villalobos and B. Aguirre-Hernández, "Necessary conditions for Hadamard factorizations of Hurwitz polynomials," Automatica, vol. 47, no. 7, pp. 1409-1413, 2011.
[20] C. A. Loredo-Villalobos and B. Aguirre-Hernández, "Hadamard factorization of Hurwitz stable polynomials," Dynamics of Continuous, Discrete \& Impulsive Systems. Series A. Mathematical Analysis, vol. 22, no. 4, pp. 325-332, 2015.

[21] B. Aguirre-Hernández, E.-C. Díaz-González, C.-A. LoredoVillalobos, and F.-R. García-Sosa, "Properties of the set of hadamardized Hurwitz polynomials," Mathematical Problems in Engineering, vol. 2015, Article ID 695279, 8 pages, 2015. 


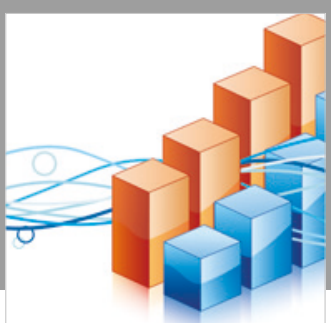

Advances in

Operations Research

\section{-n-m}
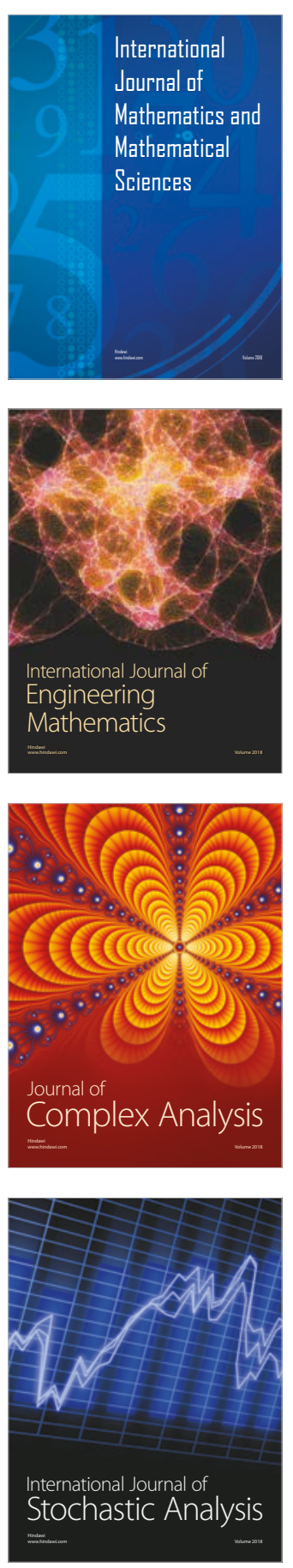
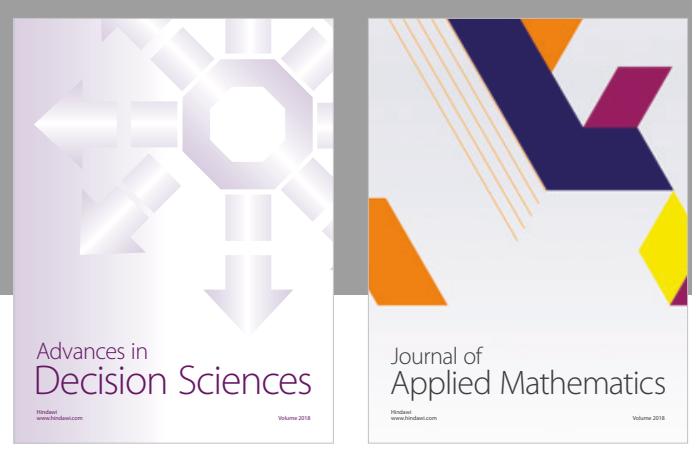

Journal of

Applied Mathematics
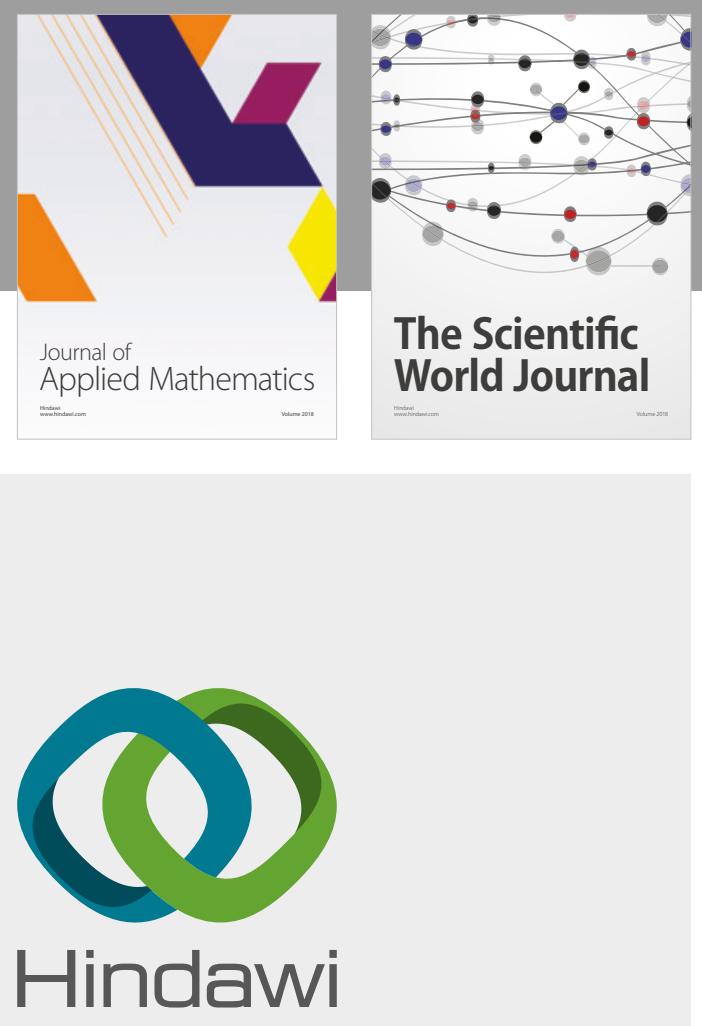

Submit your manuscripts at

www.hindawi.com

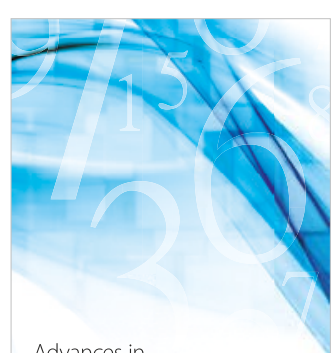

Advances in
Numerical Analysis
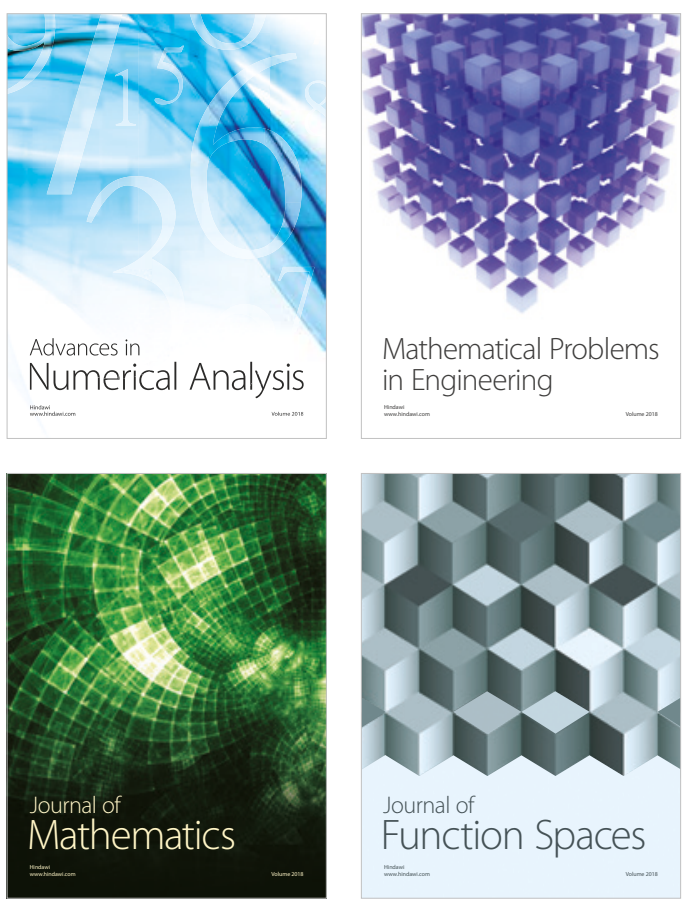

Mathematical Problems in Engineering

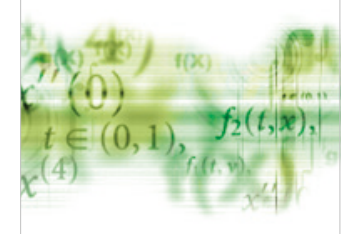

International Journal of

Differential Equations

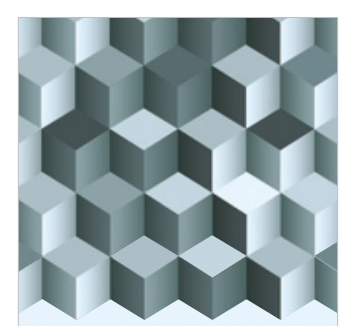

Journal of

Function Spaces

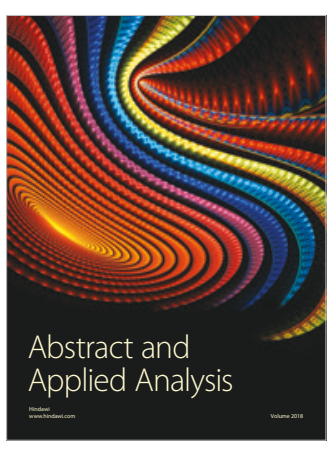

The Scientific

World Journal

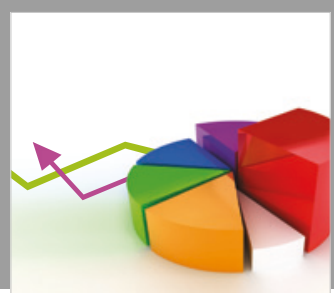

Journal of

Probability and Statistics
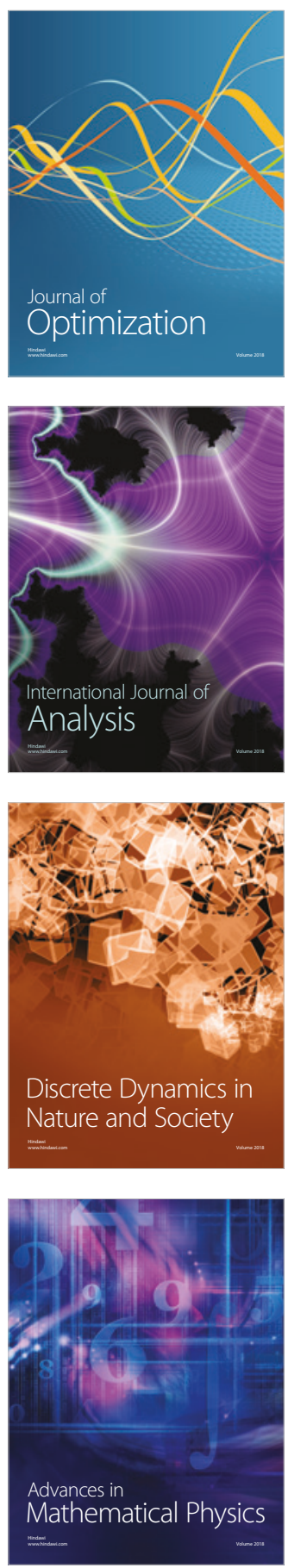